\title{
Un Code Pénal Pour l'Unité Italienne: le code Zanardelli (1889) - La Genèse, le Débat, le Projet Juridique $^{12}$
}

\section{A Criminal Code for the Unification of Italy: the Zanardelli Code (1889) - The Genesis, The Debate, The Legal Project}

Luigi Lacchè

Università degli studi di Macerata, Macerata, Itália

Resumo: O presente artigo analisa o processo de formação do primeiro código penal italiano posterior à unificação política do país, o chamado Código Zanardelli de 1889. Uma análise que contextualiza a codificação não somente na cronologia dos fatos políticos, mas também, por exemplo, na ciência do direito penal italiana do século XIX. Uma ciência do direito penal - conhecida sob o nome de "escola clássica" - caracterizada por ter levantado algumas bandeiras como a abolição da pena de morte, um dos aspectos apontados, inclusive, como fator que dificultou processo de unificação legislativa no campo penal. Por fim, a título de epílogo, são abordadas as críticas da chamada "escola positiva" que se opunha a algumas tendências liberais do recém-promulgado código. Um liberalismo penal, porém, que é visto em sua ambiguidade: no interior de um código liberal, por exemplo, dispositivos rígidos para combater o dissenso político, e uma normativa extra codicem que mitigava algumas garantias consagradas no código.

Palavras chave: História do direito penal. Código Penal Italiano de 1889. Giuseppe Zanardelli. Ciência do Direito Penal italiana no século XIX.
Abstract: This article analyzes the process of formation of the 1st Italian penal code after the political unification of the country, called Zanardelli code of 1889 . An analysis that contextualizes the encoding not only of the chronology of political events, but also, for example, in the Italian criminal law science of the XIX century. A science of criminal law - known under the name of "classical school" - characterized by having raised some flags as the abolition of the death penalty, one of the appointed aspects, inclusive, such factor that hindered the legislative unification process in the criminal area. Finally, as an epilogue addresses the criticism of the "positive school" who opposed the liberal tendencies of the newly enacted code. A criminal liberalism, however, is seen in its ambiguity: within a liberal code, for example, hard devices to combat political dissent, and rules extra codicem that mitigated some safeguards enshrined in the code.

Keywords: History of criminal law. Italian Penal Code of 1889. Giuseppe Zanardelli. The Science of the Italian Criminal Law in the nineteenth century.

${ }^{1}$ Recebido em: 24/10/2013

Revisado em: 02/12/2013

Aprovado em: 24/02/2014

2 Publicado originalmente em "Les codifications pénales: expériences, débats, enjeux (18e-20e siècle)", Actes du Séminaire histoire et justice, Bruxelles, Publications de l'Université Saint-Louis (1997). Tradução do italiano para o francês de Monique Blondel. Resumo e palavras-chave por Ricardo Sontag. 


\section{La Genèse}

Un code n'est pas seulement un corpus de normes complet et organique, élaboré par des juristes professionnels. La codification est aussi un processus culturel et historique qui correspond à des raisons politiques et à des conceptions idéologiques ${ }^{3}$.

La genèse du code Zanardelli a ses racines les plus profondes dans le vaste contexte des réformes législatives qui ont eu lieu entre le XVIII et le XIX siècle. Une analyse serait pourtant trop limitée si l'on voulait ne considérer que les aspects essentiels se référant à la chronologie institutionnelle de l'histoire italienne post-unitaire. Notre code doit, pourtant, être examiné - dans les limites d'une analyse sommaire - au sein d'un développement plus ample.

Le «problème pénal» ${ }^{5}$ est le syntagme efficace forgé pour souligner l'intensité et la centralité du débat du dix-huitième siècle sur les idéologies punitives. La «science de la législation» constitue le terrain de réflexion dans le domaine des politiques criminelles. La réforme de la législation pénale concerne le droit de punir et commence à révéler les arcana du système pénal de l'Ancien Régime dont on dénonce, pour la première fois, les privilèges, la cruauté inutile et l'inefficacité.

Dans de nombreux États italiens, le «problème pénal» représente, au cours de la seconde moitié du dix-huitième siècle, le coeur du débat culturel. L'effet Beccaria doit être vu à l'intérieur d'un phénomène beaucoup plus complexe et diversifié (des frères Verri à Paolo Risi, de Gaetano Filangieri à Mario Pagano, etc.). Le code de la Toscane de 1786 (la

3 Cf. G. TARELLO, Storia della cultura giuridica moderna. Assolutismo e codificazione del diritto, Bologna, Il Mulino, 1976, p.18 et suivantes.

4 Enrico Pessina considérait déjà l'histoire de la codification de 1889 dans une perspective plus vaste dont le 'début' pouvait idéalement coïncider avec l'oeuvre de Beccaria: Il diritto penale in Italia da Cesare Beccaria sino alla promulgazione del Codice penale vigente (1764-1890), dans Enciclopedia del diritto penale italiano, par E. Pessina, Milano, Soc. Editrice Libraria, 1906, vol. II.

5 G. TARELLO, Il «problema penale» nel secolo XVIII, dans Materiali per una storia della cultura giuridica, 5(1975), pp.15-25. 
Leopoldina) et le projet de code pénal pour la Lombardie autrichienne (1791) témoignent d'un engagement réformateur concret.

La saison des Lumières a inauguré l'idée d'une science du droit pénal pourvue d'une forte 'philosophie civile'; il s'agit d'une technique sociale qui, comme le diront d'abord Giandomenico Romagnosi, puis Pellegrino Rossi, participe à l'administration du gouvernement. Quelques expériences du XIX siècle n'ont pas trahi, dans ses lignes principales, l'héritage de la «science de la législation», c'est-à-dire de cette science nomothetica qui caractérise d'une façon particulière le XVIII siècle. La continuité des principes de garantie et de liberté formelle élaborés à l'époque des Lumières a permis de perfectionner le droit pénal du point de vue technico-dogmatique.

La science pénale, qui remonte en grande partie au XIX siècle, redonne vigueur au legs du XVIII siècle. Les criminalistes italiens jouissent, en Europe, d'un prestige considérable ${ }^{6}$. Peut-être n'y a-t-il en Italie aucune discipline juridique moderne possèdant plus de racines autonomes, profondes et prestigieuses. La science pénale, qui est une science tendant intensément vers la conception 'politique' du rapport entre le droit de punir et la société ${ }^{7}$, acquiert toute sa valeur dès l'Unité du pays. A partir de ce moment, la réflexion sur les sujets des libertés, des garanties, de la sûreté et de l'ordre atteint un caractère moderne achevé.

Le 17 mars 1861, le Royaume d'Italie est proclamé sous la dynastie des Savoie. L'Italie réalisera une unité législative presque achevée au moyen des codes (civil, de procédure civile, de procédure pénale) et des lois administratives de $1865^{\circ}$. Mais, paradoxalement, la législation pé-

6 Cf. M. T. NAPOLI, La cultura giuridica europea in Italia. Repertorio delle opere tradotte nel secolo XIX, vol.I, Tendenze e centri dell'attività scientifica, Napoli, Jovene, 1987, pp.28-30.

7 M. SBRICCOLI, La penalistica civile. Teorie e ideologie del diritto penale nell'Italia unita, dans A. SCHIAVONE (sous la direction de) Stato e cultura giuridica in Italia dalla Unità alla Repubblica, Bari, Laterza, 1990, pp. 147-232.

8 Cf. G. D'AMELIO, Pasquale Stanislao Mancini e l'unificazione legislativa nel 1859-61, Annali di storia del diritto, 1961-1962, 5-6, pp. 159-220; A. AQUARONE, L'unificazione legislativa e i codici del 1865, Milano, Giuffrè, 1965; G. ASTUTI, L'unificazione amministrativa del Regno d'Italia, dans Tradizione romanistica e civiltà 
nale est la seule à rester en dehors du dessein unitaire. En 1859, par la Loi n. 3.345 du 25 avril, le Parlement subalpin avait conféré au gouvernement les pleins pouvoirs législatifs et exécutifs en cas de guerre avec l'Autriche. Le gouvernement employa ces pouvoirs pour approuver, avec une rapidité et dans une improvisation extrêmes, trois nouveaux codes (pénal, de procédure pénale et de procédure civile), inspirés à la tradition juridique piémontaise, dont l'application fut ensuite étendue - avec des protestations et des soupçons compréhensibles (surtout en Lombardie et en Toscane) - à une partie des provinces italiennes annexées entre 1859 et 1860.

Après 1860, trois codes pénaux coexistent donc en Italie. Le code sarde-piemontais de 1859 a été étendu au Royaume d'Italie'; ce même code, modifié (par le décret luogotenenziale du 17 février), est en vigueur depuis 1861 dans les Provinces napolitaines et en Sicile; le code de la Toscane de 1853 reste en vigueur dans sa région ${ }^{10}$.

Le fait que l'Unité législative n'ait pas eu lieu dans le domaine pénal - leitmotiv du débat jusqu'au code Zanardelli - est dû principalement à deux raisons: la particularité de la codification de la Toscane, l'émergence de l'ordre public dans les provinces méridionales après l'annexion. Cette affaire représente, en effet, le parcours historique le plus vaste de l'Italie libérale face aux problèmes de l'Unification. Les classes dirigeantes subalpines essayèrent de réaliser rapidement un projet unitaire qui, cela est inévitable, 'simplifiait' la réalité plus complexe des Etats préunitaires et sous-estimait (on entendit parler, déjà à l'époque,

giuridica europea, Napoli, E. S. I., 1984, t. II, pp. 847-926; C. SCHWARZENBERG, La formazione del Regno d'Italia. L'Unità amministrativa e legislativa, Milano, Giuffrè, 1975; C. GHISALBERTI, Unità nazionale e unificazione giuridica in Italia, Bari, Laterza, 1979; ID., La codificazione del diritto in Italia, 1865-1942, Bari, Laterza, 1985.

9 En particulier aux territoires du Royaume de Sardaigne et aux territoires annexés, la Lombardie et la Vénétie, les duchés émiliens, la plus grande part de l'Etat pontifical.

${ }^{10}$ Dans un article publié (le 28 avril 1863) par le quotidien florentin le plus important, La Nazione, l'on peut lire: "La unificazione la vogliamo anche noi. Anche noi vogliamo che si faccia, riparando anche alle soverchie lungaggini delle forme parlamentari. Ma la vogliamo previo esame, previa discussione degli esperti: ma la vogliamo corrispondente ai bisogni, e agli interessi delle diverse provincie italiane" (dans A. AQUARONE, L'unificazione legislativa, cit., p. 127). 
d"'unification à vapeur") les aspects originaux et particuliers de chaque nation italienne en les insérant dans une nation unitaire qui, sous certains aspects, devait être entièrement construite, ou bien inventée. La rhétorique de l'unification législative n'était rien d'autre qu'un des grands thèmes du débat politico-idéologique concernant l'Unité italienne. Et le problème de la codification pénale met en évidence - jusqu'au code Zanardelli -, sous leurs différents aspects, les contradictions (que ce soit des partisans de la centralisation ou des "autonomistes"), les difficultés, les défis du projet d'unification et l'élaboration des stratégies de raccord entre l'Etat et le champ restreint de la société civile. L'importance accordée à l'aspect unitaire des formes institutionnelles de l'organisme de l'Etat entraîne non seulement le processus d'unification juridique et administrative, mais aussi, et surtout, le projet visant à unifier des formes sociales distinctes qui devraient être perçues dans l'Etat.

Dans sa partie générale, le code piémontais de 1859 confirme les conquêtes les plus communes du siècle des Lumières: le principe de légalité stricte, la non-rétroactivité de la loi pénale, une définition plus précise de la tentative, l'abaissement des niveaux des peines et la prévision des circonstances atténuantes. Le principe de légalité subit, néanmoins, d'importantes dérogations, dans sa partie spéciale: par exemple, à propos des délits politiques et des délits contre la tranquillité publique ${ }^{11}$.

Le code (1853) de la Toscane reflétait, grâce à sa structure moderne et originale ${ }^{12}$, une culture juridique dont l'ampleur était européenne avec des juristes de la valeur de Carmignani, Mori, Puccioni et Buon-

11 Cf. G. NEPPI MODONA, Carcere e società civile, dans Storia d'Italia, V, I documenti, Torino, Einaudi, 1973, p.1917 et suivantes; ID., Legislazione penale, dans Storia d'Italia, 2, par F. Levi, U. Levra, N. Tranfaglia, Firenze, La Nuova Italia, 1978, pp. 588-589.

12 Il faut chercher les modèles qui ont inspiré le code surtout dans la science pénale et dans les codifications allemandes. A propos des codes toscans et de la science pénale du Grand-Duché, v. M. T. NAPOLI, La cultura giuridica europea, cit., pp.190-191 et surtout les apports récents de M. DA PASSANO, Il diritto penale toscano dai Lorena ai Borbone (1786-1807), Milano, Giuffrè, 1988; ID., Il primo progetto di codice penale toscano (1824), dans Materiali per una storia della cultura giuridica, 1(1992), pp. 41-64; ID., La storia esterna del codice penale toscano (1814-1859), dans Istituzioni e società in Toscana nell'età moderna. Atti delle giornate di studio dedicate a Giuseppe Pansini, Roma, Ministero per i Beni culturali e ambientali, 1994, pp. 564-589. 
fanti. Mais, au delà de la qualité technique, l'aspect qui différenciait le code de la Toscane de celui du Piémont était que le premier ne prévoyait pas la peine de mort. Au moment de son annexion au Royaume d'Italie, cela faisait plus de vingt ans que la Toscane n'avait pas vu un bourreau à l'oeuvre $^{13}$, et, après la chute des Lorena, la peine de mort avait été définitivement abolie.

L'Unité avait fait surgir le dilemme s'il fallait supprimer la peine capitale dans le reste de l'Italie aussi ou, au contraire, en étendre l'application à la Toscane. Francesco Carrara se prononça - avec d'autres - pour la conservation d'un régime différencié, dans le but de ne pas altérer les équilibres entre les traditions nationales distinctes ${ }^{14}$. Carrara faisait remarquer que

[...] la unificazione potrebbe compromettere la sicurezza in alcune province quando le condizioni di queste fossero tali da esigere una maggiore energia di castighi, e questi si dettassero per loro più miti a cagion di riguardi ad altre province che non ne abbisognassero. E potrebbe invece compromettere la giustizia, quando per riguardo a quelle si estendessero a queste i castighi più severi dei quali esse non hanno bisogno. ${ }^{15}$

Abolir ou ne pas abolir la peine de mort devint l'argument le plus actuel lors des débats et des polémiques qu'engagèrent les juristes et l'opinion publique. L'aspiration à avoir un code unique se heurtait au grand obstacle de la question de la peine de mort.

${ }^{13}$ Cf. Statistische Nachrichten über die Todesstrafe in Toskana, mit Bemerkungen über die Wirkung der Gesetzgebung in Bezug auf diese Strafe; von einem toskanischen Rechtsgelehrten, mit einem Zusatze von MITTERMAIER, dans Kritische Zeitschrift für Rechtswissenschaft und Gesetzgebung des Auslandes, 12 (1840), pp. 223-234.

14 F. CARRARA, Se la unità sia condizione del giure penale, dans Opuscoli di diritto criminale, Lucca, Giusti, 1870, $2^{3}$ ed., vol.II, pp.7-39; ID., Sulla crisi legislativa in Italia. In risposta al quesito propostomi da S.E. il Ministro Pisanelli sulla progettata estensione delle leggi penali sarde alle provincie toscane, ivi, pp. 167-214; ID., Codicizzazione, ivi, pp. 215-230.

15 F. CARRARA, Sulla crisi legislativa in Italia, cit., p. 171. 
Au lendemain de l'Unité, Carrara avait employé l'expression crisi legislativa. Le dilemme - choisir la sécurité ou la justice - dérivait de la constatation des différences profondes qui séparaient les nouvelles provinces italiennes.

Les débats sur le brigandage méridional et sur l'abolition (ou non) de la peine de mort étaient étroitement liés, mais agissaient, l'on pourrait dire, sur deux niveaux différents. Le brigandage - qui est un phénomène très complexe - reçut une réponse répressive, selon une tradition plus ancienne ${ }^{16}$, avec des instruments extra ordinem et des procédures sommaires. La polémique sur la peine de mort concernait plutôt le code pénal, ou bien les techniques "ordinaires" de contrôle politique et social de la criminalité. La répression du brigandage déterminait une suspension des garanties constitutionnelles, lacérait le tissu du système libéral que les juristes du Risorgimento avaient espéré pouvoir édifier; tout cela ne pouvait ne pas avoir une certaine influence sur le débat, hautement symbolique, concernant la peine capitale, en lésant surtout les principes libéraux de l'Etat unitaire.

Si, en 1863, alors que l'on insistait sur le caractère exceptionnel de la situation, la loi $\mathrm{Pica}^{17}$ avait introduit un système spécial de répression du brigandage, les partisans de l'abolition de la peine de mort faisaient appel, non sans une contradiction évidente ${ }^{18}$, à la nécessité de dépasser les différents régimes pénaux en adoptant un code pénal unique.

16 A ce sujet nous renvoyons à L. LACCHE', Latrocinium. Giustizia, scienza penale e repressione del banditismo in antico regime, Giuffrè, Milano, 1988; ID., 'Ordo non servatus'. Anomalie processuali, giustizia militare e 'specialia' in antico regime, dans Studi Storici, 2 (1988), pp. 361-384.

17 Cf. F. MOLFESE, Storia del brigantaggio dopo l'Unità, Milano, Feltrinelli, 1979; R. MARTUCCI, Emergenza e tutela dell'ordine pubblico nell'Italia liberale. Regime eccezionale e leggi per la repressione dei reati di brigantaggio (1861-1865), Bologna, Il Mulino, 1980; M. SBRICCOLI, La penalistica civile, cit., pp. 173-174.

18 Il ne faut pas oublier que Pasquale Stanislao Mancini lui-même, chef de file incontesté du front abolitionniste, avait présenté en 1862, à la Chambre, un projet de loi contre le brigandage (avec une extension vaste de la peine de mort) qui faisait penser, dans quelques - unes de ses parties, au décret bourbonien du 30 août 1821. C'est justement cette attitude ambivalente de Mancini - qui peu de temps après passera à l'opposition pour critiquer les excès de la politique répressive du gouvernement italien - qui permet 
In verità sarebbe assai difficile persuadersi che la Toscana sola, dove la conservazione dell'ordine pubblico non ha bisogno di questa pena estrema, si trovi in condizioni di moralità, d'istruzione e di prosperità economica cotanto superiori a quelle del resto d'Italia da supporre nelle altre provincie quella necessità della pena di morte che ivi non esiste ${ }^{19}$.

La Chambre des Députés approuva, en 1865 (16 mars), avec une bonne majorité, la proposition Mancini (17 novembre 1864) d'abolir la peine de mort, avec, toutefois, l'exclusion des “délits punis par le Code pénal militaire ou jugés par des tribunaux militaires". Mais le Sénat, dont l'attitude était plus conservatrice, vota contre l'abolition, en interprétant l'état d'âme d'une opinion publique très préoccupée par l'élimination possible de la peine capitale. Cette impasse devait caractériser l'histoire italienne de la réforme pénale jusqu'au code de 1889. Dans cette condition, la dialectique ordre/liberté devenait, d'une façon dramatique, le banc d'essai du garantisme et de l'idéologie libérale de l'État italien ${ }^{20}$.

de bien illustrer non seulement l'état d'âme difficile, mais aussi une certaine ambiguïté des partisans plus avancé des garanties libérales.

19 Relazione della commissione della Camera sul progetto di legge per l'unificazione legislativa, 12 janvier 1865, dans A. AQUARONE, L'unificazione legislativa, cit., p. 271. Ivi, v. les interventions parlementaires (1865), en particulier le célèbre discours de l'abolitionniste Pasquale Stanislao Mancini. Cf. M. SBRICCOLI, La penalistica civile, cit., p. 163 et suivantes; M. DA PASSANO, La pena di morte nel Regno d'Italia (18591889), dans Materiali per una storia della cultura giuridica, 2(1992), pp. 341-397. A propos du personnage complexe qu'était Mancini (1817-1888), grand juriste, homme politique, plusieurs fois ministre (de l'Education Nationale, de la Justice, des Affaires Etrangères), cf. A. DROETTO, Pasquale Stanislao Mancini e la scuola italiana di diritto internazionale, Milano, Giuffrè, 1954; G. D’AMELIO, Pasquale Stanislao Mancini e l'unificazione legislativa nel 1859-61, cit.; E. JAYME, Pasquale Stanislao Mancini. Internationales Privatrecht zwischen Risorgimento und praktischer Jurisprudenz, Ebelsbach, Gremer, 1980; L. FURGIUELE, La Sinistra e i cattolici. Pasquale Stanislao Mancini giurisdizionalista anticlericale, Milano, Vita e Pensiero, 1985; les essais recueillis dans le volume Pasquale Stanislao Mancini. L'uomo, lo studioso, il politico, Actes du Colloque, Napoli, Guida, 1991.

20 Cf. L. LACCHE', La giustizia per i galantuomini. Ordine e libertà nell'Italia liberale: il dibattito sul carcere preventivo (1865-1913), Milano, Giuffrè, 1990; ID., Ordre et liberté en Italie: discipline de la détention préventive et sauvegarde de la liberté individuelle 


\section{Le Débat}

L'histoire législative du code Zanardelli est l'histoire d'un chemin tourmenté qui couvre trois décennies. A partir des années 1860, la liste des projets est plutôt longue (on en comptera, à la fin, au moins douze), elle ne convient donc pas d'en présenter ici un examen détaillé. Le débat sur le code pénal est le débat par excellence: il touche toute question concernant les idéologies punitives, les mécanismes techniques, les principes qui forment le droit pénal, les orientations de la politique criminelle de l'État unitaire. Au fil des ans, nous assistons à un processus de clarification des motifs théoriques et des solutions concrètes qui devraient animer un code libéral et moderne.

Il a déjà été question de l'émergence de l'ordre publique et de l'importance de la question de la peine capitale qui ont longtemps été un obstacle à une action réformatrice efficace. Les projets qui se suivent au cours des années sont caractérisés par une certaine continuité institutionnelle. Giovanni De Falco, qui avait élaboré, en 1864, le premier livre d'un projet de code pénal confia, une fois devenu, en 1865, ministre de la Justice, à une commission composée de juristes dont la valeur est incontestée (entre autres Francesco Carrara, Raffaele Conforti ${ }^{21}$, Pasquale Stanislao Mancini, Enrico Pessina ${ }^{22}$, Giuseppe Vacca ${ }^{23}$ et Filippo Ambrosoli ${ }^{24}$ ) la

après l'Unité, dans Entre l'ordre et la liberté, la détention provisoire. Deux siècles de débats, sous la direction de Ph. Robert, Paris, L’Harmattan, 1992, pp. 198-212.

${ }^{21}$ La personnalité politique de Raffaele Conforti (1804-1880) s'enchevêtre inséparablement avec celle du juriste, célèbre avocat napolitain, ministre du gouvernement constitutionnel napolitain de 1848, puis exilé au Piémont, député, ministre Garde des Sceaux en 1878, haut magistrat Cf. G. MONSAGRATI, s.v., dans Dizionario biografico degli italiani, Roma, 1982, vol. 27, pp. 806-810.

22 Il faut ranger Enrico Pessina (1828-1916) parmi les plus grands pénalistes de l'Italie libérale. Avocat napolitain, persécuté par la police bourbonnienne, exilé à Livourne, il fut, par la suite, professeur (Bologne), magistrat et plusieurs fois député. Sénateur en 1871, ministre Garde des Sceaux en 1885.

23 Giuseppe Vacca (1808-1876), magistrat du Royaume des Deux-Siciles, libéral, en exil en Toscane après 1850. Il fut ensuite procurateur général auprès de la Cour de Cassation de Naples, sénateur à partir de 1861, et, en 1864, ministre de la Justice.

${ }^{24}$ Filippo Ambrosoli (1823-1872), docteur en droit à Pavie (1847), participa à la première guerre d'indépendance dans un des corps de volontaires lombards; il entreprit ensuite 
tâche d'achever la rédaction du code. La commission élabora le second livre, selon un schéma préparé par Ambrosoli, et tout le projet fut présenté au nouveau ministre De Filippo en mai 1868. Ce projet fut ensuite repris en 1869 par le ministre Michele Pironti et accompagné, encore une fois, d'une relation de Filippo Ambrosoli.

Ces premiers projets doivent faire face au problème de l'héritage des traditions législatives pré-unitaires. Le débat concerne surtout l'introduction - ou non - dans le code de la discipline relative aux délits commis par la presse et le problème des contraventions. En outre, la question de la peine de mort et celle de la classification et des niveaux des sanctions.

Le thème de l'exclusivité et du primat du code, par rapport à des collocations possibles pour certaines matières en dehors du code, conserve une grande importance au cours des années soixante-dix aussi: il est possible de le constater dans le nouveau projet De Falco de 1873 ainsi que dans celui de Vigliani l'année suivante ${ }^{25}$.

En 1874, Luigi Lucchini commença à publier la Revue Pénale ${ }^{26}$, qui aura une fonction importante de proposition et de critique à l'égard du projet d'unification législative ${ }^{27}$. La Revue Pénale est la tribune de la science pénale du Risorgimento qui a élaboré le code de 1889. Le mosaï-

la carrière de la magistrature, il fut fonctionnaire du ministère de la Justice. Pénaliste cultivé, auteur, entre autres, des importants Studi sul Codice penale toscano confrontato specialmente con l'austriaco, Mantova, 1857. Cf. A. AQUARONE, s.v., dans Dizionario biografico degli italiani, Roma, 1960, vol. II, pp. 732-734.

25 Sur les premiers projets et sur les travaux de préparation, v. B. PAOLI, Storia scientifica del decennio di preparazione del Primo libro del codice penale italiano, Firenze, Niccolai, 1880; ID., Esposizione storica e scientifica dei lavori di preparazione del codice penale italiano dal 1866 al 1884. Libro secondo. Parte speciale, Firenze, Niccolai, 1885.

26 Cf. M. SBRICCOLI, Il diritto penale liberale. La «Rivista Penale» di Luigi Lucchini, 1874-1900, dans Quaderni fiorentini per la storia del pensiero giuridico moderno, 16(1987), pp.105-183, auquel nous renvoyons aussi pour les différents aspects de la biographie intellectuelle de Lucchini (1847-1929).

27 «Questa valorosa Rivista, sotto la direzione del poderoso ingegno di Luigi Lucchini, cui si deve in massima parte il progresso della scienza penale italiana dal Carrara e dal Pessina in poi, usciva con un programma nel quale era fatta principalissima parte all'idea unificatrice» (V. MANZINI, Codice penale, dans Il Digesto italiano, Torino, Ute, 18971902, vol. VII, parte II, p. 505). 
que des orientations et des attitudes scientifiques auquel on voudra donner - avec une simplification excessive - le nom d'École classique, se concentre autour des années 70 sur des thèmes étant l'objet d'une discussion générale ${ }^{28}$. Le Programma del corso di diritto criminale de Fran-

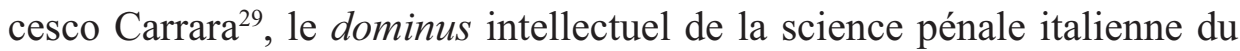
XIX siècle, constitue le moment culminant de la fondation scientifique de l'École italienne ${ }^{30}$. Le problème de la codification pénale rapproche toute une génération de juristes - malgré la diversité des vocations et des orientations - dans le débat culturel, dans les chambres, dans les commissions ministérielles,. Ce sont des juristes qui ont presque tous participé au mouvement du Risorgimento, qui ont en commun une inspiration libérale; ce sont des juristes qui - ayant été formés dans les domaines culturels des Etats pré-unitaires - côtoient assez souvent la science pénale d'études philosophiques de tendance éclectique; ce sont des professeurs qui sont souvent appelés à exercer les fonctions les plus élevées de la magistrature. Il suffit d'indiquer quelques noms: Giuseppe Vacca, Raffaele Conforti, Baldassarre Paoli ${ }^{31}$, Gian Paolo Tolomei ${ }^{32}$, Filippo Ambrosoli, Enrico

28 M. SBRICCOLI, La penalistica civile, cit., p.185 et suivantes.

29 Sur ce juriste (1805-1888) et sur son oeuvre, cf., parmi les apports les plus récents, A. BARATTA, Filosofia e diritto penale. Note su alcuni aspetti dello sviluppo penalistico in Italia da Beccaria ai nostri giorni, dans Rivista internazionale di filosofia del diritto, 49(1972), p. 34 et suivantes; M. SBRICCOLI, Dissenso politico e diritto penale in Italia tra Otto e Novecento. Il problema dei reati politici dal «Programma» di Carrara al «Trattato» di Manzini, dans Quaderni fiorentini, 2(1973), p. 567 et suivantes; A. MAZZACANE, Francesco Carrara, dans Dizionario biografico degli italiani, Roma, 1977, vol. XX, pp. 664-670; F. COLAO, Il delitto politico tra Ottocento e Novecento. Da «delitto fittizio» a «nemico dello Stato», Milano, Giuffrè, 1986, p.73; M. SBRICCOLI, Il diritto penale liberale, cit., passim; M. CATTANEO, Francesco Carrara e la filosofia del diritto penale, Torino, Giappichelli, 1988; Francesco Carrara nel primo centenario della morte, Actes du Colloque, Lucca-Pisa 2/5 juin 1988, Milano, Giuffrè, 1991; M. SBRICCOLI, La penalistica civile, cit., p.160 et suivantes.

30 F. CARRARA, I cardini della scuola penale italiana, dans Rivista Penale, 5(1876), pp. 148-163.

31 Baldassarre Paoli (1811-1889), magistrat florentin, fut fréquemment membre des commissions ministérielles qui élaborèrent le code pénal.

32 Gian Paolo Tolomei (1814-1893) fut professeur de droit et de procédure pénale à l'Université de Padoue. Il est, par exemple, l'auteur d'un Diritto penale filosofico e positivo austriaco (1863). 


\section{Pessina, Antonio Buccellati ${ }^{33}$, Tancredi Canonico ${ }^{34}$, Pietro Ellero ${ }^{35}$, Luigi Casorati $^{36}$, Pietro Nocito ${ }^{37}$, Emilio Brusa ${ }^{38}$, Giovan Battista Impallomeni, Luigi Lucchini.}

33 Antonio Buccellati (1831-1890), enseigna le droit pénal à Padoue et débuta, alors qu'il suivait l'enseignement de Carrara, avec l'oeuvre intitulée Sommi principi di diritto penale, Milano, 1865. Favorable à l'abolition de la peine de mort, il fit partie, en 1876, de la commission gouvernementale pour le nouveau code pénal. Critiquant les principes de l'école positive, il publia, à Milan, en 1884, les Istituzioni di diritto e procedura penale secondo la ragione e il diritto romano. Sur ce juriste, v. M. CARAVALE, s.v., dans Dizionario biografico degli italiani, Roma, 1972, vol. XIV, pp. 753-754.

34 L'on cite plus souvent Tancredi Canonico (1828-1908) parce qu'il adhéra à la mystique chrétienne du polonais Towianski et à son apostolat politico-religieux plutôt que pour son apport comme pénaliste (fortement inspiré par ses convictions religieuses), en tant que professeur à Turin, puis, à partir de 1876, magistrat et, plus tard, Président de la Cour de Cassation de Florence. Cf. M. THEMELLY, s.v., dans Dizionario biografico degli italiani, Roma, 1975, vol. XVIII, pp. 171-175.

35 Pietro Ellero (1833-1933), après une brève expérience comme professeur de philosophie du droit, à Milan, devint, en 1861, professeur de droit pénal à l'Université de Bologne, se distinguant par sa participation active à la campagne pour l'abolition de la peine de mort. Fondateur, en effet, du Giornale per l'abolizione della pena di morte (1861) (cf. M. SORESINA, Pietro Ellero e il dibattito sulla pena di morte (1861-1865), in Il Risorgimento, 38(1986), pp. 96-117) et, en 1868 de l'important Archivio giuridico, il ne dédaigna pas l'engagement politique (il fut plusieurs fois député, nommé sénateur en 1889) ni la tâche singulière d'écrivain moraliste et sociologue. Personnage de premier plan dans le processus d'élaboration du code pénal unitaire (de 1863 à 1888), ami de Zanardelli, il devint en 1880 conseiller de la Cour de Cassation, à Rome, et dix ans plus tard, conseiller d'Etat. Sur la longue existence d'Ellero, voir C. VANO, s.v., dans Dizionario biografico degli italiani, Roma, 1993, vol. XLII, pp. 512-520.

36 Le magistrat Luigi Casorati (1834-1885), personnage du Risorgimento, garibaldien, joua un rôle considérable dans l'oeuvre de réforme du système pénal italien. Auteur du premier grand commentaire du code de procédure pénale (1874-1881), il consacra justement une des oeuvres les plus importantes de l'école classique au procès criminel (Il processo penale e le riforme, Milano, 1881). Voir, à ce propos, P. CRAVERI, s.V., dans Dizionario biografico degli italiani, Roma, 1978, vol. XXI, pp. 422-423.

37 Pietro Nocito (1841-1904), fut d'abord professeur de droit et de procédure pénale à Sienne (1868), puis de philosophie du droit à Turin (1872), enfin, pendant une trentaine d'années (1874-1904), professeur de droit et de procédure pénale à Rome.

38 Emilio Brusa (1843-1908), étudia à Padoue, puis à Pise (disciple de Francesco Carrara). Il enseigna à Modène, puis à Amsterdam, enfin à Turin, succédant à Tancredi Canonico. Juriste libéral, s'intéressant beaucoup à la philosophie, il fut aussi fervent du droit pénal 
Ces auteurs contribuent, d'une manière différente, à la préparation du code. La conquête du pouvoir par la sinistra, en 1876, interrompt le cheminement du projet Vigliani. Le nouveau ministre de la Justice, Pasquale Stanislao Mancini, ne partage pas certains des choix de fond du projet, il propose donc une série d'amendements qui seront examinés par une commission ad hoc. A la fin de 1877, le premier livre du projet de code est approuvé par la Chambre des Députés.

Ce vote - qui abolit la peine de mort - inaugure le soi-disant régime de l'abolizione di fatto de la peine capitale qui, bien qu'elle soit prescrite et prononcée, n'est pas exécutée depuis cette époque-là. De plus, le débat à propos des différents niveaux, la modération, la certitude et sur l'efficacité des peines acquiert, au cours de ces années, de plus en plus d'importance.

$\mathrm{Au}$ cours des années suivantes, l'examen du livre II du projet de code, promu par les ministres de la Justice Conforti (1878), Tajani (18781879) et Villa (1879-1881) continua. Il faut pourtant attendre les années 80 pour voir la concrétisation de ce que Lucchini, dans sa revue, essayait de promouvoir $^{39}$. Le parlementarisme stérile devait recevoir un élan décisif de la part de l'élaboration scientifique ${ }^{40}$.

Le projet de 1883 engage, pour la première fois, le garde des Sceaux Zanardelli ${ }^{41}$. L'idéologie libérale et l'exaltation de l'unification législative caractérisent l'exposé des motifs. Le projet, qu'il faudrait plus

international et des législations pénales comparées. Cf. C. SPADA, s.v., dans Dizionario biografico degli italiani, Roma, 1972, vol. XIV, pp. 679-680.

39 L. LUCCHINI, Ai lettori, dans Rivista Penale, 21(1885), pp. 5-19.

${ }^{40}$ Cf. M. SBRICCOLI, Il diritto penale liberale, cit., p.134 et suivantes.

41 Sur l'homme d'Etat brescian (1826-1903), voir, au moins, E. ONDEI, Giuseppe Zanardelli e un trentennio di storia italiana, Brescia, 1954; G. CAROCCI, Agostino Depretis e la politica interna italiana dal 1876 al 1877, Torino, Einaudi, 1956; C. VALLAURI, La politica liberale di Giuseppe Zanardelli dal 1876 al 1878, Milano, 1967; E. SENESI, Giuseppe Zanardelli dalla giovinezza alla maturità, Brescia, 1967; L. MASCILLI MIGLIORINI, La Sinistra storica al potere. Sviluppo della democrazia e direzione dello Stato (1876-1878), Napoli, 1979; Giuseppe Zanardelli, 1826-1901, par R. Chiarini, Milano, Angeli, 1985; R. CAMBRIA, Alle origini del ministero ZanardelliGiolitti. L'ordine e la libertà, I-II, dans Nuova rivista storica, I-II (1989), pp. 67-132; V-VI (1989), pp. 609-656. 
exactement appeler Zanardelli-Savelli, vu que celui-ci lui a succédé en mai 1883, absorbe ce qu'il y avait alors de plus avancé et de plus moderne. Certaines modifications seront introduites dans les projets suivants. Mais c'est seulement à partir de 1887 - quand Zanardelli retourne au ministère de la Justice ${ }^{42}$ - qu'a lieu la phase conclusive de l'élaboration du code pénal italien.

Le texte dut être rédigé de nouveau, mais toujours suivant les principes et les contenus du premier projet Zanardelli. La rédaction du texte fut l'oeuvre de Giovan Battista Impallomeni ${ }^{43}$. Les idées apportées par Luigi Lucchini ${ }^{44}$ furent fondamentales pour le code. La Chambre et le Senat approuvèrent le projet après que les commissions l'eurent examiné et en eurent fait rapport, en 1888. Lors de l'audience du 30 juin 1889 un événement «en mémoire dans la législation nationale» ${ }^{45}-$ Zanardelli présenta le code au roi. Publié en 1889, il entra en vigueur le ler janvier 1890.

\section{Le Projet Juridique}

Le code pénal unitaire met fin à la saison tourmentée de la réforme législative. Le code Zanardelli est le code du libéralisme modéré et réaliste qui avait trouvé dans le Programma de Carrara un système de conceptions scientifiques admirable. C'est un code qui, comme le dit Lucchini, vise toujours à une idée de réalité et non pas à un «punto di vista astratto

${ }^{42}$ Zanardelli fut ministre de la Justice de trois gouvernements successifs: Depretis, 4 avril-29 juillet 1887; Crispi, 7 août 1887-9 mars 1889, 9 mars 1889-6 février 1891.

${ }^{43}$ Cf. M. SBRICCOLI, Il diritto penale liberale, cit., p.135. Giovan Battista Impallomeni (1846-1907), magistrat, attachés au Ministère de la Justice, professeur de droit et de procédure pénale à partir de 1890 (Parme, Palerme, Rome).

${ }^{44}$ V. MANZINI, Codice civile, cit., p. 511: «Se noi ricerchiamo pertanto a chi spetti il merito d'aver dato all'Italia un codice, che tanta copia di ammirazione presso di noi ed all'estero raccolse e raccoglie, non par dubbio l'affermare ch'esso spetta al Lucchini». Le premier projet Zanardelli (1883) avait été élaboré par Lucchini, Casorati, Ellero et Canonico.

${ }^{45}$ L. LUCCHINI, La terza serie della Rivista Penale, dans Rivista Penale, 31 (1890), p. 5. 
ed effimero» ${ }^{46}$. Le code est le résultat mûr d'une oeuvre collective, d'une intervention corale de la science juridique ${ }^{47}$.

L'architecture du code Zanardelli repose, comme on l'a dit, sur quelques postulats du "garantisme" libéral. Sa structure est assez moderne et fonctionnelle. L'aspect le plus remarquable et symbolique est, sans aucun doute, l'abolition de la peine de mort (et de la peine des travaux forcés). Mais nombreux sont les aspects innovants. Le code introduit le système de la bipartition (délits et contraventions), se détachant de la tradition des codes préunitaires (sauf le code de la Toscane) et du modèle napoléonien. Le code contient aussi bien les dispositions générales que les dispositions spéciales sur les contraventions pourvues d'une plus grande stabilité formelle.

Le concept de l'objectivité juridique du fait est l'élément qui qualifie la notion de crime. L'imputation ${ }^{48}$ personnelle des crimes dépend de l'individuation des critères formels. Les peines sont déterminées par le juge avec un pouvoir discrétionnaire plus grande. Les sanctions sont moins lourdes, de plus le juge peut appliquer les circonstances attenuantes. Ensuite des peines alternatives et des succédanés pénaux sont prévus. Le système des peines fait la distinction entre la réclusion et la détention suivant le type de délits, avec une différenciation du régime pénal. L'institution de la liberté conditionnelle est un des mécanismes introduits en fonction de la rééducation et de la correction du condamné.

La définition de la tentative apparaît plus articulée: le commencement de l'exécution en est l'élement constitutif. Dans un délit, la responsabilité des complices est proportionnée au rôle effectif joué par chacun d'eux. Le concours matériel des délits est réglé selon le principe du cumul juridique.

\footnotetext{
46 L. LUCCHINI, Il progetto del nuovo codice penale, dans Rivista Penale, 27 (1888), p. 11.

${ }^{47}$ Le code «rappresenta l'opera collettiva dei più dotti penalisti italiani, meditata e corretta per il corso di quasi un trentennio» (F. BENEVOLO, L'unificazione legislativa penale, Torino, 1890).

48 Sur la notion d'imputabilité, cf. E. DEZZA, Imputabilità e infermità mentale: la genesi dell'articolo 46 del codice Zanardelli, dans Materiali per una storia della cultura giuridica, 1(1991), pp. 131-158.
} 
Dans sa partie spéciale, le code Zanardelli cherche à mettre en oeuvre le système des principes-guides présents dans la partie générale. Le cas prévus mettent l'accent sur l'intérêt objectivement lésé, avec l'intention de sauvegarder les rapports juridiques et les biens déjà disciplinés et prévus par le code civil. Les normes de la partie spéciale n'arrivent pas toujours à être en cohérence avec les principes généraux. La matière des délits contre la sûreté de l'État prévoit un certain renforcement du «magistero punitivo per le forme più gravi di reati contro l'esistenza organica dello Stato e i suoi più vitali interessi...» ${ }^{49}$, mais, en même temps, elle vise à une modération majeure des peines. D'une façon générale, l'on cherche à atteindre, dans le code, un compromis honorable entre l'exigence de sauvegarder l'intégrité et l'existence de l'ordre étatique, et la manifestation libre du dissentiment politique dans les formes "constitutionnellement" garanties.

Dans la définition des délits contre l'administration publique, les types de délits semblent décrits avec plus de précision et distribués d'une façon plus logique. L'on a, en particulier, introduit une disposition vouée à décriminaliser la résistance privée aux actes arbitraires ou aux excés de pouvoir des officiers publics ${ }^{50}$.

${ }^{49}$ G. CRIVELLARI, Il codice penale per il Regno d'Italia (Approvato dal R. Decreto 30 giugno 1889, con effetto dal 1 gennaio 1890), Torino, Ute, 1890, vol. I, p. CCLVI.

${ }^{50}$ Sur le code Zanardelli, cf., en particulier, le Progetto del codice penale per il Regno d'Italia, preceduto dalla relazione ministeriale, presentato alla Camera dei Deputati nella tornata del 22 novembre 1887 dal ministro di grazia e giustizia e dei culti Zanardelli, Roma, Stamperia Reale, 1888; Relazione a S.M.il Re del Ministro Guardasigilli nell'udienza del 30 giugno 1889 per l'approvazione del testo definitivo del codice penale, Roma, Stamperia Reale D. Ripamonti, 1889; G. CRIVELLARI, Introduzione al Commento del nuovo codice penale italiano pp. III-CCCXXVI. Voir, encore, G. VASSALLI, Codice penale, dans Enciclopedia del diritto, Milano, Giuffrè, 1960, vol. VII, pp. 264-270; P. NUVOLONE, Giuseppe Zanardelli e il codice penale del 1889, dans Giuseppe Zanardelli, cit., pp. 163-182; P. UNGARI, Giuseppe Zanardelli guardasigilli e l'avvocatura, dans Giuseppe Zanardelli, cit., pp. 183-192; E. DOLCINI, Codice penale, dans Digesto delle discipline penalistiche, Torino, Utet, 1988, vol. II, pp. 275-276. 


\section{Epilogue}

Le nouveau code fut accueilli, dans l'ensemble, positivement ${ }^{51}$, et pas seulement en Italie ${ }^{52}$. En l'observant d'après les perspectives scientifiques de l'École classique, le code semblait l'expression la plus mûre des théories et des idéologies du droit pénal post-unitaire. Les problèmes et les thèmes qui étaient, depuis longtemps avancés dans les débats y avaient trouvé une systématisation organique bien étudiée. Pourtant, le point d'équilibre qui venait d'être atteint avait été immédiatement remis en discussion. Le Troppo presto $^{53}$ de Cesare Lombroso, slogan et jugement synthétique sur l'opportunité de publier un code qui ne tenait en aucun compte les revendications et les orientations de l'Ecole positive, souligne la dépendance du code Zanardelli envers les postulats de l'École classique,

${ }^{51}$ Cf. G. CRIVELLARI, Introduzione, cit., pp. CCXVII-CCXXVI.

${ }^{52}$ Le long parcours vers l'unification législative dans le domaine pénal avait suscité l'intérêt, par exemple, de Ch. LUCAS, Le projet de Code pénal italien et le rétablissement de la peine de mort en Toscane, Paris, 1874; V. MOLINIER, Études sur le nouveau projet de Code pénal pour le royaume d'Italie, Paris-Toulouse, 1879; Ch. LUCAS, Rapport verbal sur un nouveau projet de Code pénal présenté à la Chambre des députés d'Italie le 26 novembre 1883 par Savelli, Paris, 1884; J. LACOINTA, Le dernier projet du Code pénal italien, dans Bulletin de la Société générale des prisons, 1888, 7. Mais nous pouvons citer aussi, en Allemagne, les oeuvres de Liszt, Seuffert, Wahlberg, Stephan et, dans le monde anglo-saxon, de Bruce-Boston, de Lewis, etc. Parmi les différentes traductions l'on peut citer les traductions françaises (Code pénal italien promulgué le 1.1.1890..., trad. et annoté par Edmond TURREL; Code pénal d'Italie. Trad., annoté et précédé d'une introduction par Jules LACOINTA, Paris, Imprimerie Nationale, 1890) et la traduction allemande Strafgesetzbuch für das Königreich Italien. Nebst dem Einführungsgesetz vom 22.11.1888 und der Einführungsverordnung vom 30.6.1889, übertragen und erl. von Richard Stephan, Berlin, Reiner, 1890. Des oeuvres de Charles Lucas, de Victor Molinier, d'August Geyer furent publiées dans la Rivista Penale. Von Holtzendorff accueillit le nouveau code avec enthousiasme ( «Il Progetto attuale sembrami un lavoro incomparabile nella sua tecnica legislativa...», cf. G. CRIVELLARI, Introduzione..., p. CCXIX-CCXX, CCXXXVII-CCXXXVIII).

${ }_{53}$ C. LOMBROSO, Troppo presto, dans Appunti al nuovo Codice penale..., Torino, Bocca, 1889 (la première édition est de 1888). Le travail - écrit Lombroso - "fu dettato troppo presto, in otto giorni...". Mais le titre sous-entend l'idée d'une orientation scientifique inécoutée, la critique de l"'idéalisme" et du caractère "abstrait" du législateur, la nécessité d'interventions 'structurelles' (concernant la procédure, le système des prisons, la police etc.). 
ou si l'on veut, du "parti" de la Revue Pénale. C'était donc un code qui représentait, par ses principes informateurs, l'homogénéité substentielle des valeurs culturelles des pénalistes au cours des trente premières années de l'histoire italienne. Mais ce code entrait en vigueur quand, désormais, depuis une dizaine d'années, aux tendances scientifiques de l'école juridique italienne l'on commençait à adjoindre - en tant qu'alternative sur le plan de la stratégie de politique législative pénale - l'orientation, au coeur tout aussi bariolé, de la soi-disant Ecole positive. Il ne s'agissait pas seulement d'une conception empirique, "positive" des faits pénaux reliés aux sciences sociales nouvelles ou rénovées, l'anthropologie criminelle, la médecine légale, la psychologie, etc.

Sur le plan juridique - après avoir éliminé de la polémique les aspects les plus singuliers ou paradoxaux - le caractère alternatif (avec toutes ses conséquences pratiques, donc politiques) des propositions des deux Ecoles était évident en ce qui concerne la sécularisation, la responsabilité personnelle, l'imputabilité, la dangerosité sociale, la récidive, la pénalité et l'exécution des peines (polémique douceur/rigueur), la peine capitale et ainsi de suite. Au fond, la donnée la plus significative et durable du troppo presto lombrosien reside justement dans le fait que celui-ci met l'accent sur les prémisses et sur les options politico-juridiques différentes qui caractérisent les deux systèmes.

D'autre part, l'on comprend l'attitude de fermeture (plus que de liquidation pure et simple) manifestée par les rédacteurs du code Zanardelli envers les nouvelles propositions. Il s'agissait de la défense "naturelle" d'un code qui - comme on l'a dit - se voulait la synthèse d'un processus de maturation scientifique lent et difficile, ainsi que d'une vision politique déterminée. Les nouvelles tendances culturelles risquaient de bouleverser le projet unitaire et relativement cohérent du code. Il est pourtant certain que le travail de codification, bien que refusant de tenir compte des nouvelles sollicitations ${ }^{54}$, aurait dû - par la suite - tisser un dialogue, plus ou

54 Que l'on pense à la théorie lombrosienne - en contraste strident et provocateur avec l"'opera nazionale" d'unification de la législation pénale - proposant d'adopter des disciplines spéciales "per le grandi divisioni, come sarebbe l'Italia nordica e centrale; l'Italia meridionale e l'insulare: e, ben inteso, non per tutti reati, ma per quelli che più essenzialmente devono risentire l'influenza del clima, dei costumi, ecc." (Appunti al 
moins explicite, avec les instances plus significatives et scientifiquement valables de l'Ecole positive.

Les pivots techniques et idéologiques du code - l'abolition de la peine de mort, la modération des peines, la libération conditionnelle, etc. - furent critiqués et contestés par quelques partisans du front positiviste qui s'était renforcé. Sous d'autres points de vue, les profils les moins "libéraux" du code - par exemple concernant la définition des délits politiques $^{55}$ - furent critiqués. Le code sera bien vite aussi accusé de perpétuer des «injustices sociales» ${ }^{56}$.

On a dit que le code de 1889 était «un codice liberale per un'Italia che lo era ben poco» ${ }^{57}$. Les normes du code devaient, en effet, faire face à une réalité peu encourageante: des juges pas toujours fiables et pas toujours à la hauteur de la mission délicate et décisive qui leur était confiée ${ }^{58}$, un système pénitentiaire dramatiquement inadéquat ${ }^{59}$, un code de procédure beaucoup moins libéral et avancé que le code de droit pénal. Le pro-

nuovo codice penale, cit., Prefazione alla seconda edizione, p. XI). Lombroso intitule, et cela est significatif, le chapitre VI: L'Italia è unita, non unificata.

55 Cf. M. SBRICCOLI, Dissenso politico e diritto penale, cit., pp. 607-702; F. COLAO, Il delitto politico tra Ottocento e Novecento, cit.; M. SBRICCOLI, La penalistica civile..., cit., pp. 179 s., pour une mise à jour historiographique. En ce qui concerne les manques et les ambiguïtés concernant le code en matière de délits de presse, cf. F. COLAO, «La libertà moderata dalla legge»: i reati di stampa tra l'editto del 1848 e il codice Zanardelli, dans Materiali per una storia della cultura giuridica, 1991, 1, p. 75-92.

56 Un essai de Eugenio Florian aura comme titre Le ingiustizie sociali del codice penale, dans La scienza del diritto privato, 1896, 4, p. 47-55, 81-95. Au sujet de tous les différents aspects du socialismo giuridico, voir M. SBRICCOLI, Il diritto penale sociale. 1883-1912, dans Quaderni fiorentini, 1974-1975, 3-4, p. 557-642; ID., Elementi per una bibliografia del socialismo giuridico italiano, Milano, Giuffrè, 1976; ID., La penalistica civile..., cit., p. 212 s., pour une revue approfondie du rapport entre le droit pénal et la "question sociale".

57 M. SBRICCOLI, La penalistica civile..., p. 189 s.

58 «... ma è davvero deplorevole che i nostri magistrati, come dimostra la statistica, non siano entrati nello spirito di certi istituti, e non comprendano le finalità eminentemente sociali di questi, trascurando ciII da cui dipendono in massima parte i buoni effetti pratici della nuova legge...» (V. MANZINI, Codice penale..., p. 518).

59 «L'applicazione della nuova legge fu soverchiamente affrettata. Le profonde innovazioni apportate al precedente sistema penale esigevano un certo grado di sviluppo 
blème de la civilisation pénale ne pouvait être résolu uniquement - c'est ce qui avait été dit dans le rapport ministérielle - par le nouveau code. D'autre part, l'outillage policier, restitué au terrain moins visible de la loi de sûreté publique (1889), formait une "zone d'ombre" dans le système pénal: la "complaisance" du code Zanardelli trouvait en l'occurence dans cette loi un soutien répressif, valable principalement pour combattre les comportements déviants des "classes dangereuses" et, surtout, les formes d'antagonisme et d'opposition politique et sociale. La loi sur la sécurité publique attribuait à l'autorité de police le pouvoir d'émaner des dispositions (ammonitions, surveillance spéciale, résidence forcée, etc.) portant atteinte à la liberté personnelle, lésant ainsi un principe - l'universalité des garanties juridiques - qui semblait ne pas avoir de valeur pour les classes sociales les plus touchées potentiellement par les rigueurs de la loi de police. Une analyse précise de l'ambiguïté et de l'ambivalence structurelle du système pénal de l'Italie libérale ${ }^{60}$ est nécessaire pour evaluer dans toute sa complexité le rôle et la fonction que le code de 1889 a pu jouer dans le processus de réforme de celui-ci.

Ce n'est pas par hasard si la dernière décennie du siècle, traversée par de fortes tendances autoritaires ${ }^{61}$, fit exploser les contradictions qui existaient à l'intérieur du système. Au cours des années 1890 les mouvements de protestation sociale, les tumultes relatifs au ravitaillement, les nombreuses manifestations ayant une matrice politique, socialiste et radicale, révélèrent - dans certaines zones du pays - des anachro-

della necessaria riforma delle carceri, la quale ancor oggi, con grande scandalo e gravissimo danno, rimane da compiere» (ibidem, p. 512).

60 Sur ces thèmes, v. L. LACCHE', La giustizia per i galantuomini...

61 Sur la crise de la fin du siècle, en particulier, G. NEPPI MODONA, Sciopero, potere politico e magistratura 1870/1922, Bari, Laterza, 1969, p. 81 s.; U. LEVRA, Il colpo di Stato della borghesia. La crisi politica di fine secolo in Italia. 1896/1900, Milano, Feltrinelli, 1975; L. VIOLANTE, La repressione del dissenso politico nell'Italia liberale: stati d'assedio e giustizia militare, dans Rivista di storia contemporanea, 1976, 4, p. 481524; A. BOLDETTI, La repressione in Italia: il caso del 1894, dans Rivista di storia contemporanea, 1977, 4, p. 481-515; R. CHIARINI, La crisi di fine secolo in Italia, dans Annali della Facoltà di scienze politiche dell'Università di Milano, 1983, 3, p. 531-582; J.A. DAVIS, Legge e ordine. Autorità e conflitti nell'Italia dell'800, Milano, Angeli, 1988, p. 375 s.; R. CAMBRIA, Alle origini del Ministero Zanardelli-Giolitti... 
nismes, de profondes divisions sociales et politiques, des difficultés liées à l'intégration démocratique des masses populaires dans l'Etat et à l'instauration d'un régime de liberté authentique, capable de promouvoir l'ascension politique des nouveaux acteurs sociaux engendrés par le développement industriel. Le gouvernement eut systématiquement recours, jusqu'à la fin du siècle, à des instruments répressifs très durs, marquant ainsi les institutions libérales d'une orientation carrément autoritaire.

La dialectique entre le code pénal (qui contenait déjà quelques normes visant à contraster l'antagonisme social et politique) et le vaste système qui existait hors du code, qui côtoyait ou remplaçait celui-ci, devenait, dans ce contexte, encore une fois fondamental. Une observation attentive montre que le débat se déplaçait du code vers l'application de celui-ci. Luigi Lucchini l'avait bien compris car il publia, à partir de 1892, un $\mathrm{Su}$ pplément à la Revue Pénale, composés d'études illustrant le code: une espèce de relevé de son application et de son interprétation dans le contexte judiciaire. Ainsi, c'est le droit pénal jurisprudentiel - c'est-à-dire la mise en oeuvre du code - devenait le nouveau champ de bataille.

Luigi Lacchè é Professor universitário desde 1992. Jurista, historiador do direito, aluno do professor Mario Sbriccoli (1941-2005), professor titular de História do Direito no Departamento de Direito da Università degli studi di Macerata (Itália). Reitor da Università degli studi di Macerata (Itália).E-mail: lacche@unimc.it. Endereço profissional: Università degi studi di Macerata Rettorato Piazza della Torre, 8. CAP 62100 Macerata, Italia. 